Research Article

\title{
Effect of N-95 Mask on Headache among Healthcare Workers in Phase 2 of COVID- 19 Pandemic
}

\author{
Ghufran Jaleel', Deepak Malhotra', Sohrab A Khan', Abdur Raheem Khan ${ }^{3}$, Sana Parveen ${ }^{4}$
}

${ }^{1}$ Assistant Professor, ${ }^{2}$ Associate Professor, Department of Rehabilitation Sciences, School of Nursing Sciences and Allied Health, Jamia Hamdard, New Delhi, India.

${ }^{3}$ Associate Professor, Department of Physiotherapy, Faculty of Health and Medical Sciences, Integral University, Lucknow, Uttar Pradesh, India.

${ }^{4}$ Physiotherapist, Ayurvedic and Unani Tibbia College, Karol Bagh, Delhi, India.

DOI: https://doi.org/10.24321/2394.6539.202113

I $\quad \mathbf{N} \quad \mathbf{F} \quad \mathbf{O}$

Corresponding Author:

Deepak Malhotra, Department of Rehabilitation Sciences, School of Nursing Sciences and Allied Health, Jamia Hamdard, New Delhi, India.

E-mail Id:

drdeepakphysio@gmail.com

Orcid Id:

https://orcid.org/0000-0003-1413-6681

How to cite this article:

Jaleel G, Malhotra D, Khan SA, Khan AR, Parveen S. Effect of N-95 mask on Headache among Healthcare Workers in Phase 2 of COVID-19 Pandemic. J Adv Res Med Sci Tech. 2021;8(4):1-6.

Date of Submission: 2021-12-07

Date of Acceptance: 2021-12-29

\section{$\begin{array}{llllllll}\mathbf{A} & \mathbf{B} & \mathbf{S} & \mathbf{T} & \mathbf{R} & \mathbf{A} & \mathbf{C} & \mathbf{T}\end{array}$}

Background: During a pandemic, N-95 face masks are the most commonly used respirators by healthcare workers and the general public. The sustained wearing of the N-95 mask has led to physical distress and negative impacts on health, such as dizziness, shortness of breath, and headache. It is hypothesized that the headache severity of HCWs who wear the N95 mask for a lengthy period would change over time.

Methodology: Between February and April 2021, the preliminary study surveyed 112 health care providers according to the inclusion and exclusion criteria. The survey questionnaire includes four parts: (1) demographic information; (2) information about N95 use (6 questions); (3) headache-related information (10 questions); and (4) perceptions of the effect of face masks on their headache condition.

Results: A total of 112 healthcare workers (HCWs) took part in the study, including 38 men (33.9\%) and 74 women (66.1\%) with an average age of 28 years. N-95-related discomfort was experienced in $87(77.7 \%)$ HCWs, and 82 (73.2\%) had headaches. Only 13 (11.6\%) HCWs had a headache more than three times a week, while $33(29.5 \%)$ had it twice or more often. The Spearman correlation was used to evaluate the relationship between headache severity at the beginning and present, and there is a positive correlation in both stages.

Conclusion: The current study has shown that the frequency and severity of headaches in healthcare workers has increased significantly after wearing an $\mathrm{N} 95$ facemask in the second wave of the Covid 19 pandemic that originated in December 2019.

Keywords: N-95, Headache, COVID-19, PPE 


\section{Introduction}

In late December 2019, reports emerged from Wuhan, in Hubei Province, China, of a cluster of severe acute respiratory illnesses. ${ }^{1,2}$ By January 2020, the condition now known as coronavirus disease 2019 (COVID-19), attributed to the severe acute respiratory syndrome coronavirus 2 (SARS-CoV-2), had rapidly spread from Wuhan to other regions. ${ }^{3}$ As of December 2021, worldwide, 283,237,912 has reported confirmed cases of COVID-19. ${ }^{4}$

It spread rapidly throughout the world in a single year. Because respiratory droplets spread the virus, healthcare professionals must wear Personal Protective Equipment (PPE) while caring for patients. PPE includes gowns, gloves, respirator masks, face shields, goggles, mop caps, and shoe covers. During an ongoing pandemic, N-95 face masks are the most commonly used respirators by health care workers and public civilians. Prolonged use of the N95 mask causes mild discomfort, physiological and psychological burdens, and decreased work efficiency. Wearing the N-95 mask for an extended period has resulted in physical distress, including dizziness, shortness of breath, and headaches. ${ }^{5.6}$ Several studies have confirmed that headache is a common health issue due to the prolonged use of N95 masks. ${ }^{7}$

Scientific studies worldwide have revealed that headache is the sixth leading cause of disability worldwide. Headache interferes with personal, professional, and social activities. Headache impairs concentration at work and in daily life. It may impair work and time productivity and result in abnormal scalp sensitivity and pain. Additionally, it might become a source of sleep disturbances. ${ }^{6,8}$

Hypoxemia and hypercapnia are the primary causes of $\mathrm{N}-95$-related headaches. According to the study, hypoxemia and hypercapnia recover when the mask is doffed. As a result, the headache associated with $\mathrm{N}-95$ will not intensify with time. Additionally, external compression headache occurs when a strap injures the soft skull tissues. ${ }^{9,10}$ Due to the tightness of mask straps, the skin might be under a considerable measure of strain, and if headaches are due to compression, the pattern of the headaches will vary over time. It is hypothesized that the headache severity of Healthcare Workers (HCWs) who wear the N95 mask for a lengthy period would change over time (on average 4 to 8 hours a day). Thus, the objective is to determine the effect of the N-95 mask on the frequency, intensity, and severity of headaches in Phase II.

\section{Methodology}

A total of 112 participants were recruited from different hospitals in Delhi NCR based on the inclusion and exclusion criteria. Health care workers who were wearing N-95 masks, both male and female subjects, aged between 20 to 60 years, N-95 masks worn for a minimum of $4 \mathrm{hrs}$ were included in the study. Participants with a history of any mental or psychiatric illness, recent trauma leading to headache, any previous surgery leading to headache or any deformity which may have compromised the results were excluded from the study. Between February and April 2021, the preliminary study surveyed 112 health care providers, including physicians, physiotherapists, occupational therapists, and nurses. Permission was obtained verbally from the hospital and nursing home. The objective and scope of the study were communicated to subjects. Each participant signed a consent form.

Additionally, each participants' email address was requested. A screening list was implemented, and participants were considered for the study only if they fulfilled the inclusion and exclusion criteria. The questionnaire was sent to participants' email addresses, and they were asked to complete the questionnaire and submit it. If the subject did not respond, three reminders were given at a one-week interval, and the subject was dropped if he or she did not respond after three reminders. The questionnaire was divided into four sections: Part A contains demographic information (6 questions); Part B contains information about N95 use (6 questions); Part $C$ contains headacherelated information (10 questions), and Part $D$ contains the participants' perceptions of the effect of face masking on their headache condition.

\section{Data Analysis}

Statistical analysis was done using SPSS software. Descriptive statistics was used to analyze subjects' demographic characteristics and study variables.

Spearman rank correlation test was used to find the relationship between severity of headache in-between phase I and II and between frequency and severity of headache at present. Statistical significance was set at $P$ $<0.05$.

\section{Result}

A total of $112 \mathrm{HCW}$ sook part in the study (Table 1), including 38 men (33.9\%) and 74 women (66.1\%), with an average age of 28 years. Out of 112, 42 (37.5\%) HCWs used the N-95 mask for more than a year, and 52 (46.4\%) used it for approximately a year. As a daily routine, N-95 length was 4 hours in $46(41.1 \%)$ HCWs, 8 hours in 32 $(28.6 \%)$, and $>8$ hours in 15 (13.4\%).

$\mathrm{N}-95$-related discomfort was experienced in 87 (77.7\%) HCWs, and 82 (73.2\%) had headaches. Only 13 (11.6\%) $\mathrm{HCW}$ s had headaches more than three times a week, while $33(29.5 \%)$ had it two or three times a week. Among 112 HCWs, 56 (50\%) reported headache at the beginning of Phase 1, and 95 (75.9\%) in Phase 2, with moderate (31.3\%) to severe (10.7\%) intensity of pain on VAS at 2-5 and 5-8 respectively (Figure 1 ). 


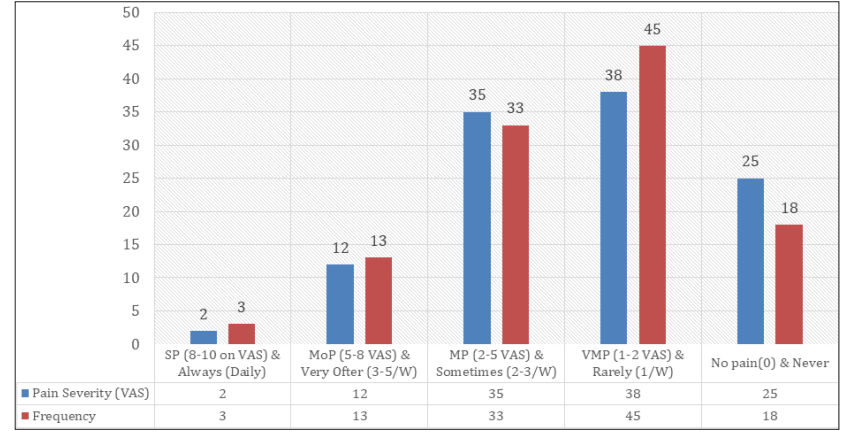

Figure I.Pain Severity and Frequency of Headache in Phase II

Only 23 (20\%) HCWs reported a sudden onset of pain, whereas 60 (54\%) reported a gradual onset, and 29 (26\%) had a varied onset of headache (Figure 2 ).

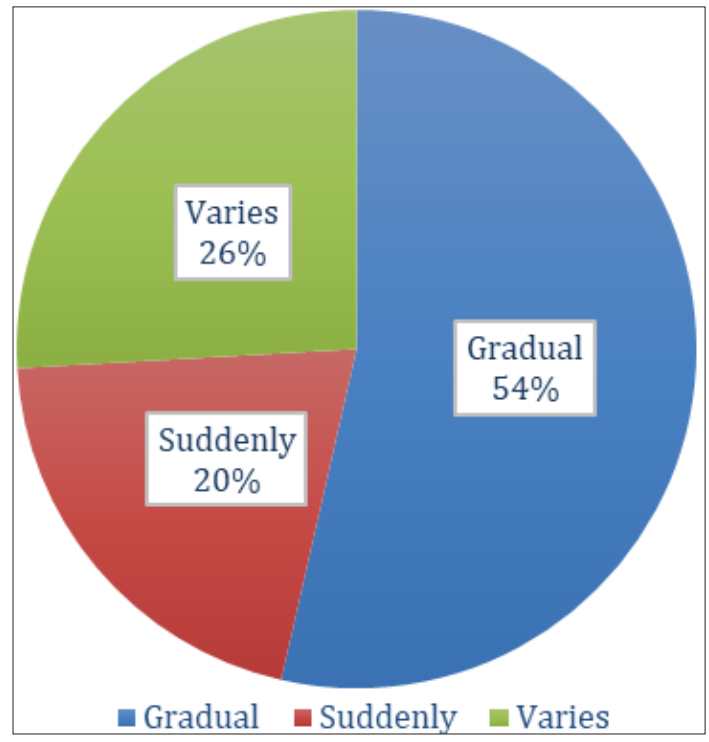

Figure 2.Onset of Headache

In 45 (40.2\%) HCWs, the pain lasted for 1-4 hours, whereas $24(21.4 \%)$ had $\leq 30$ minutes and the frequency of headache also changes as most of the HCW has 2-3 times per week (Figure 3).

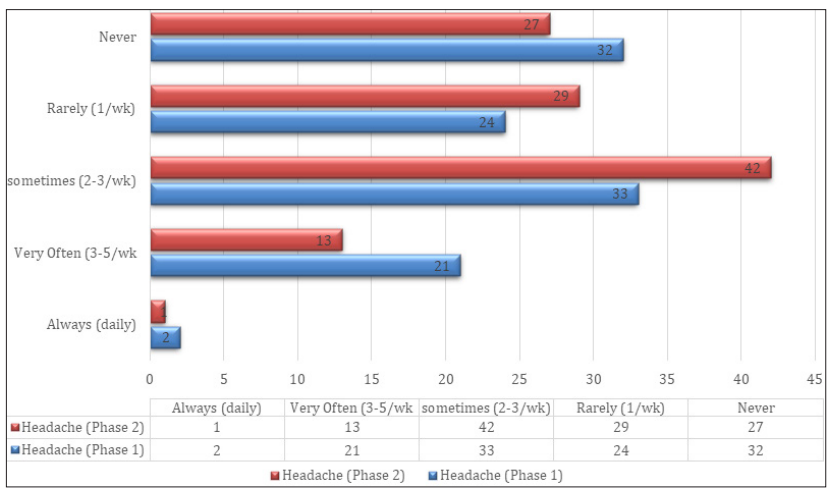

Figure 3.Change in Headache Frequency in Last one year
Among the respondents who had headaches associated with N-95, 54 (48\%) HCWs preferred or desired to lie down, and among them, 18 (16\%) always lie down, and 36 (32\%) sometimes lie down. While 43 (38\%) HCWs preferred medicine, and among them, 19 (17\%) always took medicines, and 24 (21\%) sometimes took medicines. 15 (14\%) HCWs used both the methods to relieve the pain, 41 (36.6\%) had limited usual activities at work, 47 (41.9\%) felt tired at work, 35 (31.2\%) felt irritated, and 46 (41\%) complained of limited ability to concentrate at work (Figure 4 and 5).

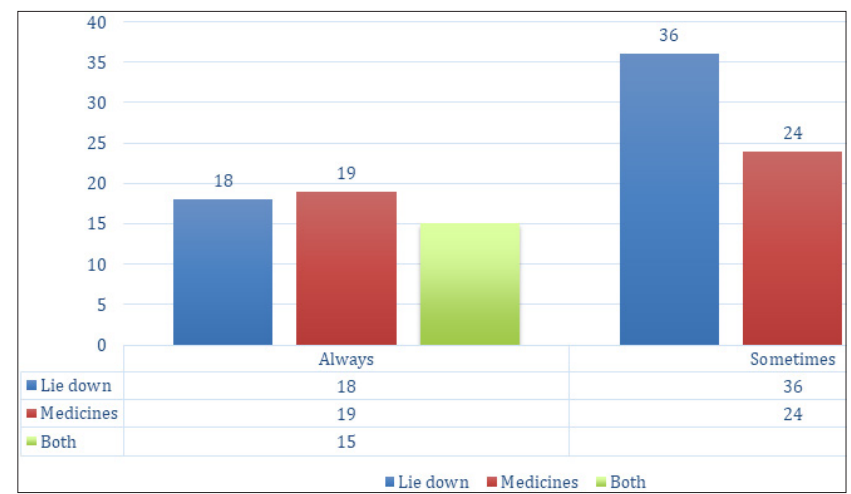

Figure 4.Pain-relieving Methods used for N-95 Associated Headache

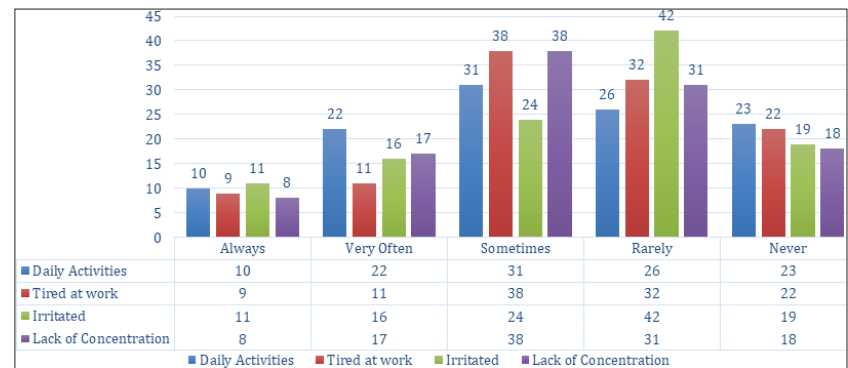

Figure 5.Effect of Headache on Physical and Mental Health

Table I.Summary of Key Feature of N-95 Associated Headache among HCWs

\begin{tabular}{|c|c|}
\hline Categories & Frequency (\%) \\
\hline \multicolumn{2}{|c|}{ Age, years } \\
\hline Mean & $28 \pm 2$ \\
\hline Range & $20-30$ \\
\hline \multicolumn{2}{|c|}{ Gender } \\
\hline Male & 38 (33.9\%) \\
\hline Female & $74(66.1 \%)$ \\
\hline \multicolumn{2}{|c|}{ Longevity of N-95 } \\
\hline$>1$ year & $42(38 \%)$ \\
\hline$\approx 1$ year & $52(47 \%)$ \\
\hline $6 \mathrm{M}$ & $9(8 \%)$ \\
\hline
\end{tabular}




\begin{tabular}{|c|c|}
\hline $3 \mathrm{M}$ & $9(8 \%)$ \\
\hline \multicolumn{2}{|c|}{ Duration of N-95/Day } \\
\hline$>8 \mathrm{H}$ & $15(14 \% \%)$ \\
\hline$\approx 8 \mathrm{H}$ & $32(29 \%)$ \\
\hline 4-6 H & $46(41 \%)$ \\
\hline $\mathrm{N}-95$ related discomfort & $87(78 \%)$ \\
\hline $\mathrm{N}-95$ related headache & $82(73 \%)$ \\
\hline \multicolumn{2}{|c|}{ Frequency of headache } \\
\hline$>3 / \mathrm{W}$ & $13(12 \%)$ \\
\hline $2-3 / W$ & $33(30 \%)$ \\
\hline $\begin{array}{l}\mathrm{N}-95 \text { related Headache } \\
\text { (phase 1) }\end{array}$ & $56(50 \%)$ \\
\hline $\begin{array}{c}\mathrm{N}-95 \text { related Headache } \\
\text { (phase 2) }\end{array}$ & 95 (75.9\%) \\
\hline \multicolumn{2}{|c|}{$\begin{array}{l}\text { Pain Severity } \\
\end{array}$} \\
\hline Moderate $(2-5)$ & $35(31 \%)$ \\
\hline Severe (5-8) & $12(11 \%)$ \\
\hline \multicolumn{2}{|c|}{ Onset of Pain } \\
\hline Gradual & $60(54 \%)$ \\
\hline Sudden & $23(20 \%)$ \\
\hline Varies & $29(26 \%)$ \\
\hline \multicolumn{2}{|c|}{ Duration of Pain } \\
\hline $1-4 \mathrm{H}$ & $45(40 \%)$ \\
\hline$\leq 30 \mathrm{Min}$ & $24(21 \%)$ \\
\hline \multicolumn{2}{|c|}{ Pain Relieving methods } \\
\hline Lie Down & $54 / 112(48 \%)$ \\
\hline Always & $18(16 \%)$ \\
\hline Sometimes & $36(32 \%)$ \\
\hline Medicines & $43 / 112(38 \%)$ \\
\hline Always & $19(17 \%)$ \\
\hline Sometimes & $24(21 \%)$ \\
\hline Always Both & 15/112 (14\%) \\
\hline
\end{tabular}

The Spearman correlation was used to evaluate the relationship between headache severity at the beginning and present, which revealed a significant positive correlation between the two stages $(r=0.343 ; p<0.05$ and $r=0.713$; $p<0.05$, correspondingly). Also, headache frequency and severity are significantly correlated $(r=0.343 ; p<0.05)$. The longevity of $\mathrm{N}-95$ masks and duration of N-95/day also showed a significant relationship with frequency and onset of headache ( $r=0.282$ and 0.229 , respectively).

\section{Discussion}

The purpose of this study was to determine the relationship between headaches and the use of an N-95 face mask by healthcare professionals throughout phases I and II of the ongoing Covid 19 Pandemic.

A total of 112 subjects, including doctors and nurses, participated in this study. Of the total subjects, about $66 \%$ were females, and $34 \%$ were males. Approximately two-thirds (68\%) of healthcare providers surveyed belong to the age category of 20-30 years old, followed by $16 \%$ who belong to 30-40 years of age. N95 masks have been used by nearly half of healthcare providers (46\%) in the last year, $38 \%$ in the previous year, and $8 \%$ in the previous 3 and 6 months. Nearly $29 \%$ of the participants wore masks for 8 hours, while $13 \%$ wore them for more than 8 hours, $17 \%$ for 6 hours, and $42 \%$ for 4 hours. 87 (78\%) of healthcare workers have reported experiencing discomfort while wearing an $\mathrm{N} 95$ mask.

More than half $(73 \%)$ of the HCWs surveyed in this study reported headaches with the $\mathrm{N}-95$ face mask, and we identified pre-existing headaches and prolonged duration of N95 face mask wear as significant risk factors for developing these headaches. The severity and frequency of headache while wearing an $\mathrm{N}-95$ mask were found to be low positively correlated with statistically significant $(r s=0.343, p<0.05)$ at the beginning and strong positive at present, which is statistically significant $(r s=0.713, p<0.05)$. Additionally, it is found that the number of healthcare workers who experienced headaches during Phase I of the pandemic increased during Phase II. The increase in headaches among HCWs in Phase II might be due to the constant compression from the straps, which is well-documented in the literature as there are conflicting views about the causative role of hypoxemia and hypercapnia in developing PPE-associated headaches. ${ }^{9,11}$ Pressure or tractional force exerted from the straps of the N95 mask may lead to local tissue damage and exert an irritative effect on the underlying superficial sensory nerve, particularly in the trigeminal or occipital nerve branches innervating the cervical and head region.

In the current survey we found that headaches developed gradually, suddenly, and with varying intensity in $54 \%, 20 \%$, and $26 \%$ of the HCWs. $54(48 \%) \mathrm{HCWs}$ preferred or desired to lie down, and among them, 18 (16\%) always lie down, and $36(32 \%)$ sometimes lie down. While $43(38 \%)$ HCWs preferred medicine, and among them, $19(17 \%)$ always took medicines, and 24 (21\%) sometimes took medicines. While 15 (14\%) HCWs used both methods to relieve the pain. The percentage of HCWs who used the medicine always for headaches is nearly half in our study compared to Ong et al. ${ }^{9}$ who found that about $32 \%$ of $\mathrm{HCWs}$ took medicine. The reason for less use of medicine could be that most of the HCWs wish to lie down to relieve the pain instead of taking medicines.

This study also accounted for the impact of headaches on daily activities while wearing an $\mathrm{N}-95$ mask in HCWs. A large 
proportion of respondents, 41 (37\%) HCWs, reported that the headache induced by the $\mathrm{N}-95$ face mask limits their everyday activities, whereas 46 (41\%) and 47 (41.9\%) HCWs felt a lack of concentration and being too tired at work, respectively, as a result of headaches. Ramirez-Moreno et al. (10) had a similar finding comprising $67 \%$ of respondents had limited physical ability and lacked the concentration to do usual work activity. The factor behind the lack of concentration and limited activity could be because of the level of anxiety or stress the HCWs faced as multiple ways of relating stress and headache have been described as 'de novo' headache. In the SARS-CoV-2 pandemic, healthcare workers may be affected by critical incident stress (CIS). Critical incidents are events in which people witness or experience tragedy, death, serious injury or threatening situations, which can have a substantial emotional impact. The signs and symptoms of CIS can be physical, cognitive, emotional, and behavioural. ${ }^{10}$ In the present study, the difference in limited physical activity and other associated side effects due to the $\mathrm{N}-95$ mask could be because of fewer subjects or the accommodation of $\mathrm{N}-95$ mask usage for an extended time.

Not many studies have been done previously to study the phenomena of headaches associated with the N-95 facemask during Covid 19. However, Lim et al. ${ }^{7}$ have conducted a similar study comprising $212 \mathrm{HCWs}$, including $22 \%$ males and $78 \%$ females with a mean age of 31 years. They reported that $37 \%$ had headaches associated with an $\mathrm{N}-95$ face mask, of which $41 \%$ of HCWs wore a mask for more than or equal to 4 hours. In the present study, most of the HCWs (47\%) wore the mask for $\geq 8$ hours while 46 (42\%) wore it for 4 hours. In the current study, however, daily use of a mask for 4 hours or more did not affect headache frequency, duration, or severity, but it did have a minor impact on the onset of the headaches ( $r=0.229, p<0.05)$. Ramirez-Moreno et al. discovered a similar result, with $47 \%$ of HCWs wearing masks for 1 to 4 hours. The minimal time could be 3-4 hours of $\mathrm{N}-95$ mask to cause headaches as physiological changes such as increased respiratory resistance were observed after 3 hours of use (10). The duration of headaches was between 1-4 hours in 45 (40.2\%) of HCWs, and $24(21 \%)$ had it $\leq 30$ minutes, as in total $69(67 \%)$ had pain $\geq 30$ minutes while approximately $95 \%$ of HCWs reported headaches of more than 30 minutes in the study by Ong et al. ${ }^{9}$

The participants who were using the N-95 mask for over one year showed a significant impact on the frequency of headaches ( $r s=0.282, p<0.05)$, and the onset of pain affected the severity of headaches $(r=0.409, p<0.05)$. The pain onset is gradual, mainly in most HCWs (54\%); only 23 (20\%) subjects complain of sudden onset of pain. It shows that the pain is slowly increasing in nature over time.

\section{Conclusion}

In summary, we found that the frequency and intensity of headaches experienced by healthcare professionals in Delhi increased significantly when they used an N-95 face mask during the Covid 19 Pandemic, which began in December 2019. While we have established this, the results may vary with the passage of time and the introduction of fresh updates.

\section{Limitations}

We acknowledge some limitations of our study. First, the sample size $(n=112)$ of the study may be considered minor, and the survey is of concise duration. However, the restriction imposed by infection control and the outbreak of COVID-19 was at a peak during that period, which made it difficult to approach the HCWs working in hospitals. Second, our study did not account for BMI, sleep disorders, nutrition, and psychological stress factors. Similarly, other factors like ambient room temperature, humidity, duty hours were not assessed. Third, our study did not assess the efficacy of the analgesics and other methods used to treat $\mathrm{N}-95$ caused headaches.

\section{References}

1. Huang $C$, Wang $Y$, Li X, Ren L, Zhao J, Hu Y, Zhang L, Fan G, Xu J, Gu X, Cheng X, Yu T, Xia J, Wei Y, Wu W, Xie X, Yin W, Li H, Liu M, Xiao Y, Gao H, Guo L, Xie J, Wang J, Jiang R, Gao Z, Jin Q, Wang J, Cao B. Clinical features of patients infected with 2019 novel coronavirus in Wuhan, China. Lancet. 2020;395:497-506. [PubMed] [Google Scholar]

2. Wu Z, McGoogan JM. Characteristics of and important lessons from the coronavirus disease 2019 (COVID-19) outbreak in China: Summary of a report of 72314 cases from the Chinese center for disease control and prevention. JAMA. 2020 [Epub ahead of print]. doi: 10.1001/jama.2020.2648. [PubMed] [Google Scholar]

3. Zhu N, Zhang D, Wang W, Li X, Yang B, Song J, Zhao X, Huang B, Shi W, Lu R, Niu P. A novel coronavirus from patients with pneumonia in China, 2019. N Engl J Med. 2020; 382:727-33. [PubMed] [Google Scholar]

4. WHO [Internet]. WHO Coronavirus (COVID-19) Dashboard [cited 2021 Dec 3]. Available from: https:// covid19.who.int/

5. Shubhanshu K, Singh A. Prolonged Use of n95 Mask a Boon or Bane to Healthcare Workers During Covid-19 Pandemic. Indian Journal of Otolaryngology and Head \& Neck Surgery. 2021 Jan 25:1-4. [PubMed] [Google Scholar]

6. Rapisarda L, Trimboli M, Fortunato F, De Martino A, Marsico O, Demonte G, Augimeri A, Labate A, Gambardella A. Facemask headache: a new nosographic entity among healthcare providers in COVID-19 era. 
Neurological Sciences. 2021 Apr;42(4):1267-76. [PubMed] [Google Scholar]

7. Lim EC, Seet RC, Lee KH, Wilder-Smith EP, Chuah BY, Ong BK. Headaches and the N95 face-mask amongst healthcare providers. Acta Neurologica Scandinavica. 2006 Mar;113(3):199-202. [PubMed] [Google Scholar]

8. Lampl C, Thomas H, Tassorelli C, Katsarava Z, Laínez JM, Lantéri-Minet M, Rastenyte D, de la Torre ER, Stovner $L$, Andrée C, Steiner TJ. Headache, depression and anxiety: associations in the Eurolight project. J Headache Pain. 2016 Dec;17(1):1-9. [PubMed] [Google Scholar]

9. Ong JJ, Bharatendu C, Goh Y, Tang JZ, Sooi KW, Tan YL, Tan BY, Teoh HL, Ong ST, Allen DM, Sharma VK. Headaches associated with personal protective equipment-A cross-sectional study among frontline healthcare workers during COVID-19. Headache: The Journal of Head and Face Pain. 2020 May;60(5):864-77. [PubMed] [Google Scholar]

10. Ramirez-Moreno JM, Ceberino D, Plata AG, Rebollo $B$, Sedas PM, Hariramani R, Roa AM, Constantino AB. Mask-associated 'de novo' headache in healthcare workers during the COVID-19 pandemic. Occupational and Environmental Medicine. 2021 Aug 1;78(8):548-54. [PubMed] [Google Scholar]

11. Ong JJ, Chan AC, Bharatendu C, Teoh HL, Chan YC, Sharma VK. Headache Related to PPE Use during the COVID-19 Pandemic. Curr Pain Headache Rep. 2021 Aug;25(8):1-1. [PubMed] [Google Scholar] 